\title{
perifèria
}

Número 18, junio 2013

http://revistes.uab.cat/periferia

\section{El silencio como metáfora. Una aproximación a la Comunidad Sorda y a su sentimiento identitario.}

\author{
Dolors Rodríguez Martín - Universidad de Barcelona ${ }^{1}$
}

\begin{abstract}
Resumen
El colectivo de personas sordas puede ser conceptualizado desde dos perspectivas dicotómicas. Por un lado la perspectiva biomédica, que entenderá la sordera como un déficit, una patología; y por otro la sociocultural, que la entenderá como una particularidad haciendo que se perciba e interprete el mundo a partir del rasgo visual. Es a partir de esta segunda perspectiva desde donde se conceptualiza la Identidad y Comunidad Sorda, siendo la Lengua de signos su rasgo identitario primordial.
\end{abstract}

Palabras clave: Sordera, discapacidad, identidad cultural, identidad sorda, Comunidad Sorda.

\begin{abstract}
The deaf people can be conceptualized from two dichotomous perspectives. The biomedical perspective that understanding deafness as a deficit, pathology. And the sociocultural perspective that understanding deafness as a peculiarity that makes the person perceives and interprets the world from the eyesight. It is from this second perspective from which identity and Deaf Community are conceptualized. In this conceptualization, sign language is the primary identity trait.
\end{abstract}

Keywords: Deafness, Disability, Cultural Identity, Deaf Identity, Deaf Community.

\section{Introducción}

Este artículo pretende ser una síntesis del extenso trabajo de investigación realizado entre 2002 y 2011, sobre el sentimiento identitario en la Comunidad Sorda. En la actualidad mi investigación continúa ahora como tesis doctoral (en la

\footnotetext{
${ }^{1}$ Departamento de Enfermería Fundamental y Médico-Quirúrgica, Escuela Universitaria de Enfermería, Campus de Ciencias de la Salud de Bellvitge, Universidad de Barcelona, España.

Enviar correspondencia a: Dolors Rodríguez Martín dolorsrodriquezmart@ub.edu

Realizando Tesis Doctoral en el programa de Doctorado de Antropología Social y Cultural de la Universidad Autónoma de Barcelona.
} 


\section{perifèria \\ Número 18, junio 2013 \\ http://revistes.uab.cat/periferia}

Universidad Autónoma de Barcelona), explorando también aspectos de salud y de toma de decisiones en la Comunidad Sorda.

En el año 2002 la lectura de un artículo del diario El País, Los sordos defienden su silencio (Alcaide 2002), hizo que el hallazgo del tema de investigación me surgiera a modo de quiebra, como mencionaría Agar (1992). En el citado artículo se describe el caso de un matrimonio de personas sordas de Madrid que acaba de tener un hijo sordo y que, ante este hecho, manifiestan una enorme alegría ya que su hijo sería "como ellos". La expresión de alegría por parte de estos padres con sordera, ante la sordera de su hijo es lo que me ocasionó la quiebra. No podía entender que unos progenitores conocedores en primera persona de lo que supone vivir con una discapacidad, pudieran desear y alegrarse de que su progenie también la tuviera y además remarcar el hecho con la expresión "sordo como ellos", marcando una frontera entre oyentes y no oyentes.

Tirando del hilo me dirigí a la fuente que suscitó el artículo anteriormente citado. Ese artículo no era fruto del azar, sino que venía precedido de otro, publicado por el mismo diario unos días antes (González 2002) en el cual se hacían eco de un artículo publicado por el Washington Post el 31 marzo de 2002 (Mundy 2002). Dicho artículo, presentaba el caso de una pareja de lesbianas que padecían sordera y que querían inseminarse con esperma de donante sordo, para que su progenie también lo fuera.

Este artículo de Mundy generó mucha controversia y muchísimas voces se alzaron poniendo el grito en el cielo: ¿cómo unos progenitores pueden desear que sus hijos/as nazcan con una carencia, con una discapacidad? Pero lo más grave no es ese deseo, sino la manipulación deliberada para que ese nuevo ser nazca sordo. Muchos críticos (Mills 2002; Cándido 2002) se preguntan cómo alguien puede atreverse a buscar una discapacidad. Otros autores, como Sandel (2007) se plantean qué es lo que resulta cuestionable: la misma elección de las características genéticas del hijo/a o las características elegidas. Todas estas preguntas se formulan desde una perspectiva biomédica, que entiende la sordera como un déficit. Pero al margen del debate en sí, me encontraba ante dos perspectivas muy diferentes y antagónicas de entender qué era la sordera: la perspectiva de las 


\section{perifèria}

Número 18, junio 2013

http://revistes.uab.cat/periferia

ciencias biomédicas o asimiladas (medicina, psicología y logopedia, principalmente) las cuales entienden la sordera como proceso clínico-patológico, incidiendo y entendiendo la misma como déficit de la persona; y la perspectiva socio-cultural (presentada por los protagonistas de los artículos) que entiende la sordera como una particularidad que hace que se perciba e interprete el mundo a partir del rasgo visual (Johnson y Erting 1989), rasgo que en la persona sorda es vivido como eje central para la interpretación y funcionamiento en el mundo.

Esta perspectiva sociocultural era nueva para mí, ya que mis conocimientos sobre el tema se limitaban a la visión hegemónica e imperante en las sociedades occidentales: la biomédica.

A partir de este momento inicié toda una serie de lecturas de acercamiento al tema y configuré la premisa de partida en la investigación:

Dentro del colectivo de personas sordas existe un subgrupo/subcolectivo usuario de la lengua de signos, que reivindica una identidad diferenciada con una cultura y lengua propia que se encuentra en oposición a la sociedad oyente mayoritaria y que se autodenomina Comunidad Sorda.

Y un interrogante cómo planteamiento de investigación:

¿Esta visión de Comunidad Sorda, como grupo lingüístico-cultural minoritario diferenciado, es compartida por el conjunto de personas que conforman esta Comunidad?

\section{Metodología}

El diseño de la investigación fue eminentemente exploratorio, desarrollándose del 2002-2011.

Las técnicas de investigación fueron: observación no participante (anotaciones de campo en diario de campo), entrevista no focalizada y semiestructurada (algunas con utilización de intérprete en Llengua de Signes Catalana), análisis documental (tanto fuentes primarias como secundarias).

Realicé 15 entrevistas, con una media de duración de 75 minutos (entre 29 minutos 


\section{perifèria}

Número 18, junio 2013

http://revistes.uab.cat/periferia

y 140 minutos).

Previa a la selección de las personas a entrevistar, confeccioné una tipología que pudiera ejemplarizar lo más ampliamente posible al subcolectivo de personas sordas. Partí de las relaciones familiares para su confección, y relacionando a ego según fuera sordo/a u oyente, y según fuera hijo/a y/o progenitor.

Otro grupo fueron las organizaciones/asociaciones del colectivo de personas sordas, tanto de orientación signista (usuarias de lengua de signos) como oralista (no usuarias de lengua de signos).

Para mostrar desde diferentes vertientes el tema, consideré incluir también la visión que tienen los/as intérpretes en lengua de signos, que actúan como puente entre la llamada sociedad oyente y la sociedad sorda.

\section{La Sordera desde el discurso biomédico y el concepto discapacidad}

Sin profundizar en aspectos fisiopatológicos, la medicina utiliza sordera como término general para describir todos los tipos y grados de pérdida auditiva. Tanto sordera, hipoacusia, como discapacidad auditiva se utilizan como sinónimos para hacer referencia tanto a pérdidas auditivas leves como profundas (Moreno 2002); otro término también utilizado, que se suma a los anteriores, es el de deficiencia auditiva.

Es importante señalar que cuando mencione sordera, hipoacusia o alguno de sus otros sinónimos, lo haré para referirme a los casos bilaterales, ya que en las sorderas unilaterales la audición es prácticamente normal, no produciendo trastorno del habla o del lenguaje, ni los inconvenientes sociales que de ello se derivan (Villalba 2001).

Desde la perspectiva biomédica, cuando se habla de sordera inevitablemente estamos hablando de discapacidad, ya que ésta deriva de la primera.

Discapacidad es un término relativamente reciente que hace referencia a la alteridad anormal, a cómo se configura y denomina a la persona que se encuentra fuera de la norma, fuera del patrón de normalidad establecido por la sociedad. La 


\section{perifèria}

Número 18, junio 2013

http://revistes.uab.cat/periferia

definición de este otro anormal variará según el contexto histórico-políticoeconómico-social en el cual nos hallemos, ofreciendo una visión determinada sobre el fenómeno. Así podemos encontrarnos ante un/a: anormal, raro, deficiente, minusválido, incapacitado, sub-normal, impedido, retrasado... más recientemente discapacitado, persona con necesidades especiales, persona no-estándar. Toda esta terminología adoptada para denominar al anormal, contrasta con la utilizada para nombrar al normal o como dice Allué (2003), al válido que resulta ser escasa.

Los diferentes términos, expresiones y clasificaciones utilizados para denominar a la anormalidad responden a diferentes fórmulas culturales, las cuales mediante una metodología que no es neutra y que "nos remite a debates ideológicos y de orden político" (Allué 2003:29), las elabora.

La concepción biomédica de la discapacidad es una construcción social relativamente reciente, pero convive con otras de carácter más antiguo (cronológicamente hablando), como la religiosa, o más reciente la social. A pesar de esta coexistencia, Allué apunta que estas diferentes visiones se mantienen "en condiciones y contextos diferentes, como mecanismos de control social de la diferencia." (Allué 2003:35).

En el siglo XIX el discurso biomédico empieza a interesarse por la conceptualización de la alteridad anormal. A partir de entonces, esta alteridad se conceptualizará como patológica, siendo el cuerpo médico el experto sobre el tema: definirán, clasificarán, diagnosticarán esta anormalidad; se encargarán de tratar y rehabilitar las pérdidas sensoriales, físicas y/o psíquicas (Allué 2003). Este modelo a su vez también será adoptado por otros sectores profesionales, tanto de las ciencias biomédicas, como de otras disciplinas (terapeutas ocupacionales, profesionales de la educación) los cuales colaborarán con el estamento médico (Allué 2003) en el tratamiento y rehabilitación de esta patología.

En los años ochenta del siglo XX irrumpe el Disability Movement, movimiento social motivado por la defensa a favor de los derechos de las personas con discapacidad, teniendo su germen de origen en los años '60, enmarcado en la movilización por los derechos civiles de las minorías. Este movimiento iniciado en EEUU y Reino Unido 


\section{perifèria}

Número 18, junio 2013

http://revistes.uab.cat/periferia

es crítico con el discurso biomédico y con la individualización de la discapacidad. A partir de este momento surge un nuevo modelo de interpretación, un modelo social que concebirá la discapacidad como resultado de la opresión social (Allué 2003).

Coetáneamente a esta perspectiva social, diferentes organismos internacionales proponen diversas definiciones y clasificaciones de la discapacidad, entendiéndola desde diferentes perspectivas: la OMS (1980) realizará su Clasificación Internacional de Deficiencias, Discapacidades y Minusvalías desde la perspectiva biomédica; la British Disabilitv Discrimination Act (1995) sin enfoque biomédico, pero sí individual (Allué 2003); la Union of the Physically Impaired Against Segregation, la definirá como forma de opresión social.

\section{Construcción social de la sordera}

La evolución histórica de la concepción de la persona con sordera ha ido en paralelo a la concepción de la alteridad anormal, en la actualidad llamada discapacidad. Pero a diferencia de otros tipos de anormalidades, la sordera y en concreto la adquisición del habla como efecto o consecuencia de la misma se han conceptualizado de manera particularizada.

Hay que matizar que la conceptualización de la persona sorda como anormal, hace referencia al "sordo de nacimiento", es decir, aquella persona que nace sorda o adquiere la sordera a tan temprana edad, que aún no ha adquirido habla (prelocutivos). Estos eran los llamados sordomudos. Las personas que la adquirían a lo largo de la vida, ya habiendo adquirido el habla (postlocutivos), no se las categorizaba de la misma manera.

Así vemos que a la persona sorda se la ha conceptualizado y construido socialmente a partir de las premisas ideológicas imperantes en el momento, sirviendo de caldo de cultivo para pensar en la persona que la padece como:

- Carente de razón y/o de alma (desde Grecia clásica hasta el siglo XIX) debido a su falta de habla o sea mudez. Se pensaba que eran personas incapaces de poder razonar, asumiendo así el precepto de la imposibilidad de recibir formación. Además, como sujetos carentes de juicio y razón, eran 


\section{perifèria}

Número 18, junio 2013

http://revistes.uab.cat/periferia

excluidos de los derechos civiles de herencia, título, matrimonio...

- Personas enfermas (desde el siglo XIX hasta actualidad), portadoras de patología. En este momento la persona sorda pasa a ser objeto de las ciencias biomédicas que la conceptualizan en función de esa patología, siendo sujetos pasivos sobre los que aplicar tratamiento y rehabilitación.

Construcción ideológica que imprime su carga simbólica sobre el binomio normal/anormal, sirviendo de descripción asimétrica entre las personas normales (oyentes) y anormales (sordas). Distinción basada en el simple hecho de no percibir sonido y en épocas anteriores, por la imposibilidad de hablar (mudez).

En el momento en que se percibe a la persona sorda como susceptible de ser educada $^{2}$, se marca el inicio de lo que posteriormente será la logopedia y la educación especial. El objetivo fundamental de esta formación académica era que la persona sorda deviniera en normal y esto sólo era posible si adquiría el habla. Para alcanzar este objetivo se han ido aplicando, a lo largo de la historia, diferentes metodologías ubicadas en la categoría de oralistas. Estas tienen como objetivo que la persona con deficiencia auditiva tenga competencia en la lengua oral, que la persona hable. Con la irrupción de la perspectiva biomédica se inician nuevas metodologías oralistas apoyadas en ayudas técnicas (que intentaran potenciar los restos auditivos) que han ido proporcionando los sucesivos avances tecnológicos. El objetivo continúa siendo el mismo: la adquisición del habla pero desde un planteamiento curador-rehabilitador.

En el extremo opuesto a estas metodologías, tenemos las de orientación bilingüe (ya que en el Estado español nunca ha habido una metodología signista pura). Estas parten de la utilización de la lengua de signos, como lengua vehicular, para en primer lugar facilitar el desarrollo cognitivo de la persona y en segundo lugar facilitar y posibilitar el aprendizaje en la lengua oral (hablada o, si más no, escrita). Pero, ¿cómo a partir de esta peculiaridad surge un sentimiento identitario? ¿Cómo se generará comunidad y cómo consecuencia de ella, cultura?

\footnotetext{
${ }^{2}$ Motivado inicialmente por la necesidad de determinadas familias nobles castellanas (s.XVI) de que su herencia y títulos no se perdieran debido a su descendencia sorda.
} 


\section{perifèria}

Número 18, junio 2013

http://revistes.uab.cat/periferia

En esta conceptualización de la sordera como anormalidad, se entiende que la persona que la padece es portadora de un estigma: "atributo profundamente desacreditador" (Goffman 2006:13). Este atributo resulta ser "una indeseable diferencia" (Goffman 2006:14) ante la cual la sociedad olvida los restantes atributos de la persona. La sociedad sólo percibirá esa diferencia, que será la que determine esa anormalidad y en consecuencia hará que se actúe con una actitud determinada, ya sea rechazo, de completa ignorancia o de paternalismo, todas ellas actitudes derivadas de esta concepción asimétrica.

Pero, ¿cómo se percibirá a sí misma la persona portadora del estigma de la sordera? Por un lado, vemos que se percibirá como normal, con las mismas ilusiones y anhelos que cualquier otra persona. Por otro lado (ya sea en situaciones vis-a-vis o en otras interrelaciones sociales) vemos que percibirá ese otro yo: el estigmatizado. Pero este hecho, que en algunas situaciones ha podido generar vergüenza (p.ej. a signar en público), no reconocimiento de la sordera (intentando por todos los medios que su voz no le descubriera, que su audífono no se notara,...) e incluso odio hacia esta circunstancia, se torna en un momento determinado orgullo (Goffman 2006).

Para llegar al sentimiento de orgullo estas personas compartirán carrera moral (Goffman 2006), una serie de experiencias similares, generadas por el estigma, las cuales efectuarán una serie de modificaciones en la concepción de su yo que implicarán cambios a nivel de identidad social y personal. Pero el hecho de compartir una carrera moral se efectuará en el contacto entre iguales. Pero para poner de manifiesto que se comparte una carrera moral es imprescindible que estas personas se relacionen entre sí, interactúen entre ellas y sean conscientes de esa realidad compartida. El lugar donde se produce este hecho han sido los centros educativos/residencias y las asociaciones de sordos/as. Es aquí donde, por primera vez el/la Sordo/a ${ }^{3}$ percibe que no está solo, que no es una persona aislada, se

\footnotetext{
3 Para diferenciar la doble visión con respecto a la persona sorda, utilizaré sordo con minúscula para hacer referencia a la deficiencia auditiva (visión biomédico) y Sordo (visión sociocultural) con mayúscula para referirme a la persona sorda que se siente miembro de una comunidad diferenciada en oposición a la sociedad mayoritaria oyente, la Comunidad Sorda. Woodward (1972) acuña esta distinción.
} 


\section{perifèria}

Número 18, junio 2013

http://revistes.uab.cat/periferia

siente entre sus semejantes, encuentra un lugar donde poner de manifiesto su normalidad, esa normalidad que le ha sido arrebatada desde la sociedad mayoritaria. Aquí empieza a gestarse ese sentimiento identitario, que hace que el grupo reaccione hacia la concepción que de él se tiene. Es aquí donde surge el sentimiento de comunidad: Comunidad Sorda. Así, la carrera moral de la persona, como proceso, ya no será estigmatizante, sino normalizadora.

Son en estos lugares (centros educativos/residencias y asociaciones) donde la persona sorda asumirá que es Sordo/a, produciéndose así una resocialización o alternación (Berger y Luckman 2001). Mediante el proceso de alternación, las personas sordas se identificarán con esa carrera moral que comparten con otras. Esta autoindentificación adquiere un carácter positivo, transformándose en identidad y en sentimiento colectivo de pertenencia a un determinado grupo.

Junto a este proceso, tanto individual (de la persona sorda), como colectivo (de los/as sordos/as agrupados en centros educativos y asociaciones), se empieza a conceptualizar a la persona sorda desde una perspectiva sociocultural (inicio 60s s.XX), perspectiva que teorizará sobre el colectivo de personas sordas. Adoptando esta perspectiva irrumpirán los Deaf Studies los cuales servirán como motor ideológico a la Comunidad Sorda para reivindicarse como minoría lingüísticocultural. Comunidad Sorda que reclamará bajo este paradigma su existencia a modo de grupo étnico, con una cultura, lengua e historia determinada, buscando su lugar en las sociedades oyentes cada vez más "multiculturalizadas".

\section{De la persona sorda al Sordo: Deaf Studies.}

La concepción patológica persiste y convive en mayor o menor armonía con nuevas concepciones de la realidad social sorda. Concepciones que ponen el acento en una interpretación de esa realidad, con base biológico-orgánica, muy diferente a la patológica que deviene en discapacidad. Estas nuevas construcciones han ido configurando un nuevo individuo sordo. 


\section{perifèria}

Número 18, junio 2013

http://revistes.uab.cat/periferia

En los años cincuenta y sesenta del siglo XX empiezan a aparecer estudios provenientes de la psicología y psicolingüística, mostrando una visión opuesta a la biomédica. Estos se centrarán más en las implicaciones de la sordera en el desarrollo general del individuo, buscando tanto similitudes como diferencias con el individuo oyente. Se analizarán aspectos de: desarrollo cognitivo-lingüístico, personalidad, estrategias de acceso a la lecto-escritura. Estas líneas de investigación también tuvieron su influencia en la educación recibida por los/as niños/as que padecían sordera, sobre todo en los planteamientos educativos que, a partir de los años setenta (ejemplo lo tenemos con los métodos bilingües), empiezan a incorporar sistemas visuales complementarios a la lengua oral (DíazEstébanez et al. 1996).

Todos estos ámbitos de estudio empiezan a explorar otros aspectos de la vida del colectivo de personas sordas: cómo se organizan como comunidad, cómo generan identidad... Al principio son apuntes (en formato de apartado de capítulos) o anexos a estas obras y, posteriormente, empiezan a suponer un nuevo campo de estudio.

Es así como aparecen los Deaf Studies. Estos hacen referencia al corpus teórico que se ha elaborado y se elabora desde la perspectiva del agente implicado, los/as Sordos/as. Hasta este momento, los estudios que se realizaban, tanto fuesen de carácter biomédico, social, lingüístico,..., no contemplaban esta visión del "nativo"4. Desde el campo de los Deaf Studies se pretende captar esta mirada de los otros/as Sordos/as, ya sean ellos/as mismos/as los/as agentes investigadores o no, pero proporcionando espacios en la academia para la participación activa y efectiva de los/as Sordos/as en el proceso de producción de conocimiento (Leigh 2009).

Todas estas aportaciones teóricas nos mostrarán cómo se configura conceptualmente la Comunidad Sorda, aportando a toda esta construcción nuevos conceptos (deaf way, audism, deafhood...) e incorporación de otros (colonialismo, deafnicity -adaptación de etnicidad-, minoría lingüístico-cultural,...) que ya han sido

\footnotetext{
${ }^{4}$ Refiriéndome aquí a la visión emic, la producida dentro de la misma comunidad; o sea, por personas Sordas. Entenderé la dicotomía etic/emic en su sentido canónico. Para un debate sobre esta dicotomía véase: González (2009).
} 


\section{perifèria}

Número 18, junio 2013

http://revistes.uab.cat/periferia

utilizados por las ciencias sociales. El nexo común de todas estas nuevas aportaciones es la perspectiva que adoptan estos estudios: la perspectiva sociocultural.

\section{Precedentes}

En los años sesenta, diferentes investigadores del campo de la lingüística y psicolingüística empiezan a interesarse por las lenguas de signos. Este es el caso de Stokoe, lingüista que investigará la American Sign Language. Con la publicación de su obra Sign language structure (1960) es el primer autor que afirma el estatus lingüístico de la lengua de signos, poseyendo las mismas características lingüísticas que las lenguas orales. Esta obra marca un antes y un después en los estudios sobre el colectivo de personas sordas, dando un paso adelante hacia la configuración de la llamada Comunidad Sorda, proporcionando entidad de lengua a lo que con anterioridad era denominado mímica. En 1965, Stockoe, Casterline y Groneberg en A Dictionary of American Sign Language on Linguistic Principles, hacen una primera descripción (en un capítulo) de las características socioculturales de las personas Sordas que utilizan el lenguaje de signos. Esta sería una de las primeras descripciones, desde sectores académicos, de la Comunidad Sorda. La lengua se torna el concepto clave que abrió el camino a los Deaf Studies, para introducirse en el discurso público en el ancho campo del movimiento por los derechos civiles y los estudios culturales (Bauman 2008).

Es importante mencionar que a pesar de que no es hasta los años sesenta que empieza a despertar el interés por los Deaf Studies, ya en los años cincuenta se empieza a mostrar a nivel internacional que existe un colectivo de personas sordas que se encuentran organizadas en sus respectivos países. Estos grupos se encuentran aglutinados en diferentes asociaciones que a su vez conforman federaciones y que mantienen contacto entre ellas. Como ejemplo, encontramos el Primer Congreso Mundial de Sordomudos celebrado en Roma en 1951, donde se fundó la World Federation Deaf (WFD). La WFD irá adquiriendo protagonismo a lo largo de los años en cuanto a sus manifestaciones y recomendaciones con respecto al tratamiento que se ha de tomar de la Comunidad Sorda. Todas estas, manifestaciones y recomendaciones, influenciadas por las aportaciones teóricas 


\section{perifèria}

Número 18, junio 2013

http://revistes.uab.cat/periferia

efectuadas desde los Deaf Studies.

Una de las primeras aproximaciones a la definición de Comunidad Sorda la encontramos en Schein (1968). Schein la definió como el conjunto de personas que son deficientes auditivas, haciendo referencia únicamente al rasgo de la discapacidad. Posteriormente esta definición será ampliada por otros autores como Baker y Padden (1978) que hacen hincapié en diferenciar más claramente los conceptos de comunidad y cultura, introduciendo el término de "sordera actitudinal" que hace referencia a la persona sorda que se identifica a sí misma como miembro de la Comunidad Sorda y que los otros miembros aceptan que forme parte de esa comunidad. Más tarde, Padden (1989) entiende que los miembros de la Cultura Sorda se comportan de manera similar, usan la misma lengua (valor cultural primordial), comparten creencias sobre sí mismos/as y sobre el resto de personas que no son Sordas (1989). La lengua de signos, además, será su lengua primaria de intercambio social, aunque posean competencia en lengua oral.

A Padden se le hacen muchas críticas, quizás la más importante es que su trabajo es poco ortodoxo y objetivo", debido a que "emplea su propia intuición y experiencia como sorda hija de padres sordos $y$, por tanto, inmersa en la Cultura Sorda desde nacimiento" (Díaz-Estébanez et al. 1996:9).

Kyle y Allsop (1982) y Kyle (1990) realizarán las primeras descripciones de las actitudes y modos de vida del colectivo de personas sordas en el Reino Unido, visibilizándolo a la sociedad oyente. Kyle (1990), además, enfatiza el papel de las asociaciones como elementos fundamentales en la vida comunitaria, aunque no toda la Comunidad Sorda acuda a ellas. Habla de ellas como "el corazón del pueblo de las personas Sordas". Es uno de los primeros autores que hablará de las reglas de conducta $^{6}$ de la Comunidad Sorda, sus costumbres, tradiciones y manifestaciones externas.

\footnotetext{
${ }^{5}$ Véase: Turner (1994).

${ }^{6}$ En el Estado español véase: Pinedo (1981).
} 


\section{perifèria}

Número 18, junio 2013

http://revistes.uab.cat/periferia

Kyle (1990) también introduce el término de Identidad Cultural, como el exponente de los factores internos que hacen que una persona se considere "parte de" una comunidad, se sienta cómoda con ella, comparta sus tradiciones y costumbres.

En 1972 Woodward acuña la distinción entre D/deaf y d/deaf (Sordo vs sordo), para distinguir la construcción cultural de la circunstancia médica y que posteriormente Paden y Humphries en 1988 adoptan, haciéndola extensible a otros autores. A partir de este momento se empieza a utilizar esta distinción en infinidad de estudios surgidos desde la perspectiva sociocultural. Sin embargo, se debe mencionar que esta distinción no ha sido adoptada de modo general (por ejemplo en la literatura en español y otros países europeos). Únicamente en inglés es posible encontrarla usada de modo coherente, sobre todo a partir de mediados de 1980 (Morales 2009).

En 1977, Humphries en su tesis doctoral "Comunicating across cultures (deafhearing) and language learning", presenta el término audism ${ }^{7}$ (audismo) para hacer referencia a una actitud discriminatoria, en la que alguien se considera superior por su capacidad de oír o de comportarse como persona oyente. Pero no es hasta 1992 que Lane retoma el término y hace extensible su uso.

Padden (2003) afirma el carácter ideológico del término (al igual que racismo, sexismo, eugenesia,...) y lo relaciona con el colonialismo cultural que se ha ejercido sobre la Comunidad Sorda. La dinámica del audism toma principalmente el carácter de la relación colonial (Bauman 2008). Lane (1992) y Ladd (2003) exploran los paralelismos entre el colonialismo y la experiencia Sorda, a través de la erradicación de las lenguas indígenas (en este caso, las lenguas de signos), educación, valores e historia de la Comunidad Sorda.

Relacionado con audism aparece hearization, término que acuña Nover (1995) para reflejar la promoción institucional, el mantenimiento de la lengua oral y los valores de la sociedad oyente. Esto crea una percepción de que las personas oyentes son superiores, y por lo tanto actúa como una forma de opresión internalizada.

\footnotetext{
7 Para ampliar véase: Bauman (2004).
} 


\section{perifèria}

Número 18, junio 2013

http://revistes.uab.cat/periferia

Otras líneas de trabajo, muy interesantes pero en las cuales no profundizaré, son las llevadas a cabo por autores como Anderson (1994) y Bahan (1994) proponiendo examinar cómo se definen las personas sordas desde sí mismas, en lugar de definirlas desde las etiquetas de Comunidad Sorda y Cultura Sorda que surgen desde los círculos académicos.

Aparición del término Deaf Studies

En 1971 se menciona por primera vez el término Deaf Studies. Fue el director ejecutivo del National Association of the Deaf, en EEUU, Frederick Schreiber haciendo referencia a que si ya existían Black studies, Jewish Studies, entonces ¿por qué no referirse a Deaf Studies? (Bauman 2008). Posteriormente, en los años setenta, Panara en 1974 publica un artículo, ya hablando de ellos: "Deaf Studies in the English Curriculum".

A partir de estos momentos se crean multitud de grupos de investigación en diferentes universidades estadounidenses y británicas (Boston University, California State University, Gallaudet University, University of Bristol). El primer grupo fue fundado por Stokoe, en la Gallaudet University, Linguistic Research Laboratory. Paralelamente, también surgen revistas especializadas como Sign Language Studies que introducen en sus artículos aspectos sociológico-culturales de la lengua de signos (Bauman 2008).

Una vez aceptada la existencia de una Comunidad Sorda, los debates dentro de los Deaf Studies se centrarán en cómo se han de desarrollar estos estudios interrogándose sobre quién es el investigador/a más adecuado: ¿̇los propios protagonistas? o ¿investigadores/as externos a la Comunidad Sorda? (Anderson 1994; Bahan 1994, 2004; Ladd 2003). Empiezan a aparecer autores/as Sordos/as iniciándose en lo que podríamos denominar auto-etnografía (Baker y Padden 1978; Padden 1989). Se categoriza la Comunidad Sorda como minoría étnica y por tanto la necesidad de abordar su estudio desde esa perspectiva ${ }^{8}$. Todos estos planteamientos que ya son tratados y analizados desde posicionamientos

\footnotetext{
${ }^{8}$ Véase: Batterbury, Ladd y Gulliver (2007); Mcllroy y Storbeck, (2011).
} 


\section{perifèria}

Número 18, junio 2013

http://revistes.uab.cat/periferia

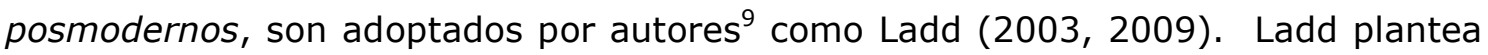
la importancia del colonialismo para el análisis de la exclusión y opresión de la Comunidad Sorda como minoría cultural; de la deconstrucción de conceptos como Hearing-Impaired, deaf; de la investigación por parte de investigadores subalternos,... Todo ello necesario para el análisis de la Comunidad Sorda.

Ladd (2003) también acuña el término Deafhood ${ }^{10}$. Deafhood hace referencia a la experiencia colectiva compartida de ser Sordo/a y de formar parte de la Comunidad Sorda. Concepto dinámico que presenta un proceso continuo de ser, que además incluiría una cultura, la Cultura Sorda, y una lengua, la Lengua de Signos. Este dinamismo presenta a la Comunidad Sorda desde la perspectiva histórica de sus logros pasados y hacia dónde se dirige en la actualidad. Según Ladd (2003) la Comunidad Sorda ha sido duramente diezmada por el oralismo y explica que la historia de los últimos 120 años $^{11}$, de gran influencia oralista, no es el reflejo de lo que realmente es la Comunidad Sorda. Para él la Comunidad Sorda está tomando conciencia de su condición y empieza a elaborar propuestas para cambiarla. Por ese motivo, mantiene que ésta debe entenderse a partir de una dimensión política, para llevar a cabo ese cambio. Y para poder desarrollar esa senda se hace necesario que se ubique todo ese proceso en lo que él ha acuñado como Deafhood.

A partir de esta nueva perspectiva de estudio, desde el Center of Deaf Studies de la Universidad de Bristol, se empieza a hablar de Deafhood Studies, invitándose a cambiar de Deaf Studies a Deafhood Studies.

Otra de las obras recientes del campo de los Deaf Studies es $A$ Lens on Deaf Identities (2009) de Leigh. Nos presenta cómo las personas sordas e hipoacúsicas forman su identidad. Esta formación es analizada desde los debates surgidos sobre qué se entiende por persona sorda, desde una perspectiva biomédica o bien

\footnotetext{
9 Uno de los primeros en utilizarlo fue Lane (1984), véase también, entre otros, Bahan y Bauman (2000); Bauman (2008).

${ }^{10}$ Algunos autores en castellano traducen como Sorditud, véase: Torres (2011).

11120 años marcados por el oralismo, remontándose a 1880: Congreso Internacional de Educadores de Sordos celebrado en Milán, en el cual la mayoría de autores dicen que fue la declive de Comunidad Sorda con la obligatoriedad del método oralista en la educación.
} 


\section{perifèria}

Número 18, junio 2013

http://revistes.uab.cat/periferia

sociolingüística. La importancia del libro viene por el tratamiento que hace la autora sobre el tema de la identidad, ya que no lo contempla como algo estático y monolítico ${ }^{12}$, sino como un concepto dinámico, en constante transición que es influida por factores como son: la audición, familia, experiencias educativas, elección tipo de comunicación (lengua oral, lengua de signos), habilidades comunicativas, historia de contacto entre Sordos/as y sociedad oyente, discapacidades secundarias, relaciones de género, orientación sexual, origen étnico, influencias y experiencias sociales, la tecnología y la moderna medicina génica.

Las aportaciones que se han hecho desde el Estado español a los denominados Deaf Studies son relativamente escasas. Como antecedentes, podemos mencionar las obras de Pinedo $(1981,1989)$. En ellas nos introduce en el mundo de la sordera y de las personas que la padecen. Son obras escritas de manera autobiográfica en las cuales hace un retrato (sin ningún afán científico sino más bien vivencial) de: cómo viven, cómo se relacionan, en qué dificultades se pueden encontrar las familias ante el diagnóstico de la sordera, cómo educar a un niño/a sordo/a,... Resultan dos obras qué a mi juicio, retratan una época juntamente con los acontecimientos vitales que repercutieron en la vida de las personas sordas, además de pretender ser un libro de ayuda a los progenitores que tienen un hijo/a sordo/a. Libros que se introducen en temas, como la sexualidad (incluida la homosexualidad), que no se habían tocado con anterioridad tan directamente (evidentemente bañados con las concepciones y argumentaciones propias del momento en que fueron escritos).

Una fecha importante en el seno de la Comunidad Sorda en el Estado español son las jornadas organizadas por la CNSE en Madrid (19-22 marzo 1992), Jornadas sobre Nuestra identidad: iiSordos!!, el progreso está en nuestras manos. A ellas acudieron personas relevantes de la Comunidad Sorda transnacional quienes explicaron: los valores, creencias, actitudes de las personas sordas, su lengua, cultura, historia e Identidad Sorda. Estas conferencias marcaron un antes y un después en cuanto al autoconcepto del colectivo de personas sordas signistas, autodenominándose desde ese momento Comunidad Sorda. Entre los asistentes y

\footnotetext{
${ }^{12}$ Como ejemplo véase: Ladd (2003)
} 


\section{perifèria}

Número 18, junio 2013

http://revistes.uab.cat/periferia

organizadores también se generó un gran sentimiento colectivo y ansias por luchar por lo que les era propio, por recuperar su identidad.

A partir de este momento, empiezan a llegar las influencias teóricas del mundo anglosajón y empiezan a producirse las primeras obras, o bien traducciones de anglosajonas, editadas por la $\mathrm{CNSE}^{13}$. También desde otros ámbitos, como el educativo, el psicológico o el lingüístico se empiezan a hacer descripciones del colectivo de personas sordas signantes como Comunidad Sorda (Marchesi 1993; Fernández-Viader, Pertusa, 2004). La CNSE empieza a editar, también, libros sobre personajes ilustres de la historia de la Comunidad Sorda, ya fueran oyentes (como maestros) o sordos. Y empieza a aparecer algún libro en formato novela autobiográfica ${ }^{14}$.

Paralelamente, se producen otras obras de carácter historiográfico de autores como Storch de Gracia y Gascón. Obras que pretenden mostrar una versión más fiable y menos sesgada, según los autores, de la historia de la educación sorda y de los personajes ilustres (oyentes y/o sordos) de la Comunidad Sorda. Según estos autores, la CNSE influenciada por las corrientes anglosajonas, han dado por válidas fuentes poco documentadas, creándose así mitos historiográficos que se elaboran en aras de la creación de una Historia Sorda unificada y segregacionista, exaltando los episodios opresivos a los cuales ha estado sometida la población sorda a lo largo de los tiempos.

\section{Conclusiones}

\section{Las organizaciones sordas signistas definen a la persona que padece sordera desde la perspectiva sociocultural.}

A pesar de ello no participan estrictamente de esta perspectiva, ya que en función de las necesidades e intereses también participaran como miembros de otras instituciones $\mathrm{y} / \mathrm{o}$ asociaciones que representan al colectivo de personas con

\footnotetext{
${ }^{13}$ Véase: Moreno (2000).

${ }^{14}$ Véase: Cedillo (2004), Martínez et al. (2007).
} 


\section{perifèria}

Número 18, junio 2013

http://revistes.uab.cat/periferia

discapacidad. Estas organizaciones a pesar de mantenerse en el discurso sociocultural, admiten que existe una discapacidad auditiva, pero que este hecho no te hace ser discapacitado, ya que el/la Sordo/a en su vida no se siente así. La persona Sorda, refieren, no tiene problemas por no oír, sino por las barreras que la sociedad oyente, que es la mayoritaria, pone a la hora de comunicar y transmitir la información, que no les llega.

La persona Sorda corriente adopta en cierta medida el discurso biomédico pero negando la asimetría entre normalidad/anormalidad.

La persona Sorda define la sordera como discapacidad, pero haciendo hincapié en que es una discapacidad auditiva. Lo que pretenden remarcar es que su supuesta discapacidad no es equiparable al resto de discapacidades, ya que el problema principalmente radica en las barreras de comunicación que son impuestas desde la sociedad oyente.

La Identidad Sorda, promulgada por los organismos que representan a las personas sordas, es percibida por los/as Sordos/as de maneras muy diversas.

Las personas Sordas entrevistadas no daban importancia al discurso sobre la Identidad Sorda. Comentaban que como cualquier otra persona, su identidad no respondía sólo al hecho de ser Sordo/a (haciendo una clara referencia a la identidad personal). Para ellos/as era ya un hecho la sordera, y como consecuencia su identidad se veía influenciada por ello, pero no era lo que principalmente les definía. A pesar de esta apreciación, que siempre se comentaba al iniciar la pregunta, sí que había un sentimiento de orgullo (en algunos casos más evidente que en otros) del hecho de ser Sordo/a.

Pero en la construcción del sentimiento identitario hay factores que influyen:

Familia de origen:

- Sordos/as hijos/as de oyentes: el sentimiento se empieza a gestar en contacto con otros/as Sordos/as (centros educativos/residencias, asociaciones en los casos más tardíos,...). Cuando se inicia este contacto 


\section{perifèria}

Número 18, junio 2013

http://revistes.uab.cat/periferia

todos/as tienen la sensación de haber encontrado su lugar, un espacio y un mundo donde no existen barreras comunicativas.

- Sordos/as hijos/as de Sordos/as: sentimiento vivido de una manera más sosegada, natural, asumido desde la cuna. No tienen necesidad de hacer bandera de este hecho, ya que saben que son Sordos/as porque su familia es Sorda. Su mundo es el Mundo Sordo y sus relaciones con el mundo oyente son marginales (trabajo y lugar de residencia). Así mismo, en algunos casos afirman su nulo interés en el mundo oyente, a pesar de que algunos de estos/as Sordos/as tienen hijos/as oyentes.

- Hijos/as oyentes de progenitores sordos (CODA ${ }^{15}$ : su lengua materna es la lengua de signos, participan de la Comunidad Sorda (son parte de ella), pero en la mayoría de los casos se ubican en el mundo oyente, aún sin olvidar la Comunidad Sorda.

Centros educativos/residencias y asociaciones son lugares de gran influencia para la configuración de la identidad Sorda. Esto se debe fundamentalmente al contacto entre iguales, no existen las barreras comunicativas (se rompe el aislamiento), se sienten libres de las miradas del mundo y es donde encuentran referentes adultos Sordos (sobre todo en el caso de los/as hijos/as de progenitores oyentes).

En relación con el nivel cultural, el/la Sordo/a, que muchas veces resulta ser un/a analfabeto funcional, con un nivel cultural bajo debido a una formación en la cual primaba el hecho de que hablara igual que "un loro"16 (horas y horas de rehabilitación logopédica), en detrimento de una acción formativa integradora de conocimientos, sabe y se siente Sordo/a, orgulloso/a de serlo pero sin entender e incluso nunca haber oído el término Identidad Sorda.

La lengua de signos es considerada por los organismos cómo eje fundamental sobre el cual se articula y fundamenta la Identidad Sorda, la Comunidad Sorda y además genera una cultura concreta.

\footnotetext{
${ }^{15}$ CODA: Child Of Deaf Adults.

16 Término literal que algunos entrevistados utilizaban para mencionar el habla sin integración de conocimiento.
} 


\section{perifèria}

Número 18, junio 2013

http://revistes.uab.cat/periferia

Por ello la consideran patrimonio de la Comunidad Sorda. En cambio los/las Sordos/as corrientes lo que enfatizan es la importancia que tiene a la hora de la comunicación, sin plantearse si es o no generador de cultura.

Para los organismos e instituciones, la lengua de signos ha sido el caballo de batalla para reivindicar la Comunidad Sorda como minoría lingüístico-cultural. Bajo esta visión, fomentada, sustentada y adoptada de los Deaf Studies, la lengua genera cultura.

Pero esta visión no es compartida por el/la Sordo/a corriente. La importancia que le dan a la lengua es una importancia más práctica y cotidiana, como: facilitadora de la apertura al mundo, comunicación, posibilitadora de expresión de sus sentimientos, herramienta para salir de su aislamiento y mediadora para abrir el camino a la información y adquisición de conocimiento.

Las personas sordas, a pesar de no mencionar directamente la vinculación de la lengua de signos con la creación de una minoría lingüístico-cultural (que se propugna desde los organismos e instituciones), sí que consideran que esta es su lengua, la lengua de las personas Sordas, su lengua natural, se sienten orgullosos/as de ella y viven con entusiasmo el reconocimiento que se le ha dado por parte de la sociedad oyente. Reconocimiento materializado en la aprobación de la Ley $27 / 2007^{17}$.

A pesar de que se pueda pensar lo contrario, los/as Sordos/as dan también mucha importancia a la lengua oral. Ellos/as viven en un mundo oyente: sus familias, mayoritariamente son oyentes; su trabajo, principalmente, se desarrolla entre oyentes; la información se transmite en lengua oral (hablada o escrita), y por lo tanto creen necesario su conocimiento. Pero lo que sí reclaman, es que la formación sea bilingüe, que se aprenda la lengua oral a través de la lengua de signos.

17 LEY 27/2007, de 23 octubre, por la que se reconocen las lenguas de signos españolas y se regulan los medios de apoyo a la comunicación oral de las personas sordas, con discapacidad auditiva y sordociegas. 


\section{perifèria}

Número 18, junio 2013

http://revistes.uab.cat/periferia

\section{Las personas Sordas tienen conciencia de que pertenecen a un grupo, una comunidad, aunque en algunos casos lo denominan más como Mundo Sordo.}

La Comunidad Sorda es construida (desde organismos e instituciones) en oposición a la sociedad oyente mayoritaria, pero los/as sordos/as corrientes no la definen en función de una oposición binaria, sino más bien en el uso o no uso de la lengua de signos. El centro de este mundo son las asociaciones, lugar donde desarrollan su vida social y en algunos casos también la laboral.

La Comunidad Sorda es concebida como un pueblo (a nivel de ubicación geográfica ${ }^{18}$ ) y el centro de este pueblo se ubicaría en las asociaciones. En las asociaciones será donde se desarrolle la vida social de los/las Sordos, en ellas: se reúnen, establecen relaciones sentimentales, realizan actividades de acción política, realizan actividades lúdicas y formativas... Y al igual que en los pueblos, también existen: cotilleos, peleas, enemistades,... También es curioso observar que entre diferentes asociaciones, sobre todo de la misma ciudad, existe rivalidad que puede crear pequeños roces entre sus habitantes.

\section{Los/as Sordos/as perciben y reconocen su Mundo, su Comunidad, pero no todos/as están de acuerdo en que derivado de ello exista una Cultura Sorda.}

El término Cultura Sorda es a veces un término muy abstracto para los/las Sordos/as, que se manejan mejor, a la hora de la comprensión del término, con los componentes de esta cultura. Consideran que tienen: costumbres propias; historia propia, la cual se ha ido mostrando desde las asociaciones y demás organismos Sordos (conferencias, libros,...); tradiciones (el día del Sordo/a); producciones culturales (teatro, cine, poesía...) aunque algunos/as Sordos/as consideran que son iguales a las del mundo oyente; y también comparten valores (a pesar de no saber concretar cuáles son).

\footnotetext{
${ }^{18}$ Desde las federaciones utilizado pueblo como sinónimo de Comunidad Sorda, dando a entender así su categoría como minoría lingüística-cultural, que además es transnacional.
} 


\section{perifèria}

Número 18, junio 2013

http://revistes.uab.cat/periferia

\section{El/la Sordo/a conoce sus organizaciones y hasta cierto punto las reconoce como Estado de su Comunidad, reconociendo así la existencia de una comunidad transnacional.}

Desde las asociaciones se realiza la labor de difusión de los modelos marcados desde las instituciones/organismos que representan a las/los Sordos/as: definición y pertenencia a la Comunidad Sorda, definición de lengua de signos, sociedad oyente, Cultura Sorda, métodos educativos más adecuados,... Estas asociaciones serían la base (pueblos o ciudades) de la Comunidad Sorda, que a su vez formarían parte de su federación autonómica, a modo de gobierno autonómico. De hecho, y como me mencionan en alguna entrevista, estas federaciones autonómicas son las responsables de realizar las oportunas gestiones y/o reclamaciones, a su respectivo Gobierno Autonómico, que tengan que ver con la Comunidad Sorda de cada autonomía. Después estas federaciones se agrupan en la Confederación Estatal (CNSE) la cual actuaría como Estado de la Comunidad Sorda española. Pero a su vez nos encontramos con dos organismos más, que serían internacionales y que nos configurarían la Comunidad Sorda transnacional. Estos organismos son: la European Union Deaf y la World Federation Deaf.

Los/las Sordos/as están de acuerdo en que para ellos/as es más fácil entablar relación con otro/a Sordo/a extranjero que con el/la vecino/a oyente del quinto. La comunicación es más fácil a pesar de tener lenguas de signos diferentes, dos días de contacto y ya pueden tener una comunicación fluida, cosa que no sucedería entre oyentes. Pero además sienten que se encuentran entre hermanos/as compartiendo los mismos problemas y anhelos, en definitiva se reconocen como miembros de la misma comunidad, miembros de la Comunidad Sorda transnacional.

Desde una concepción de Comunidad Sorda como minoría lingüísticocultural, asemejada a grupo étnico, existen ejemplos de grupos y personas Sordas que llevan al límite esta visión.

Dos ejemplos de ello serían:

Laurent City: Marvin Miller, impulsor del proyecto, es Sordo Egregado de la 


\section{perifèria}

Número 18, junio 2013

http://revistes.uab.cat/periferia

Gallaudet University, que dirige el diario Deaf Nation, el más leído por la Comunidad Sorda estadounidense. En 2005 proyecta la creación de una ciudad (en Dakota del Sur) para Sordos/as, donde la lengua oficial será la American Sing Language.

- Contre la proposition de loi dépistage précoce de la surdité ${ }^{19}:$ grupo surgido en Facebook en respuesta a proposición de ley por parte del Gobierno francés para la realización de cribados de detección precoz de la sordera en infantes. La argumentación que se da, por parte de este grupo, es que esta ley sólo beneficiará a determinados sectores de la biomedicina, además de suponer un ataque frontal contra la Comunidad Sorda francesa.

Estos ejemplos ilustran una noción de Comunidad Sorda como grupo étnico minoritario y en riesgo de exterminio. Noción en absoluto compartida por la mayoría de la Comunidad Sorda corriente, pero que sí se adopta, en determinados momentos, por los organismos e instituciones que les representan. Estos organismos apelan al sordocidio (en palabras de Storch), como exterminio, alertando del peligro de perderse la esencia de esta Comunidad y Cultura, que es la lengua de signos.

\section{A pesar de que en el pensamiento en la Comunidad Sorda parece darse un seguidismo de la concepción homegeinizadora de las organizaciones que les representan, existen voces críticas a estos modelos.}

Estas voces críticas tanto son desde el Sordo/a corriente, como de determinadas asociaciones de Sordos/as que en un momento determinado discrepan de su federación y/o federaciones que discrepan de la Confederación Estatal.

Estas voces disonantes pretenden romper, según mencionan, con la visión hegemónica, totalizadora y homogeinizadora de los discursos que pretenden definir y explicar cuál es la realidad Sorda. Desde estas críticas se defiende la libertad individual del/la sordo/a a definirse como sordo/Sordo, al igual que la libertad de utilización o no de la lengua que decida. Son aspectos muy criticados, ya que si se

\footnotetext{
${ }^{19}$ Véase: http://www.facebook.com/depistage.surdite (consultada: 16.05.2013)
} 


\section{perifèria}

Número 18, junio 2013

http://revistes.uab.cat/periferia

quiere participar de las asociaciones, que son el corazón del pueblo sordo, se ha de sentir como Sordo/a (con Identidad Sorda) y hablar en su lengua. Además los entrevistados, que son críticos con estas nociones, comentan que desde los organismos se inician guerras con respecto a los términos, y en cambio se olvida al/la sordo/a (sea con mayúscula o no), sus verdaderas necesidades (alfabetización, trabajo, integración en la sociedad).

Al margen de todo lo concluido, tras este largo periodo de investigación, de lo que no hay duda es que la sordera no deja de ser un déficit fisiológico. El ser humano tiene una serie de capacidades (como especie) que le permiten interaccionar con el medio y sus semejantes. Si falta alguna de estas capacidades, como es el caso de la funcionalidad auditiva, la interacción de este ser humano con el medio y sus iguales variará. Pero de ahí a incurrir en que este hecho proporciona un déficit en la persona, déficit entendido como esencia de la misma y que la desplaza a una posición de inferioridad con respecto a sus semejantes, es otra cuestión. Lo evidente es que, como consecuencia a esta peculiaridad sensorial, la persona se organizará y organizará su vida. Organización que se realizará básicamente en torno a la experiencia visual, la cual le permitirá casi en exclusiva interaccionar con el mundo que le rodea y generar un marco de interpretación e interacción determinado, también fruto de esta peculiaridad.

\section{Bibliografía}

Agar N. (1998). Hacia un lenguaje etnográfico. En: Geertz C., et al. (1998) El surgimiento de la Antropología Posmoderna. Barcelona: Gedisa, 117-137.

Alcaide S. (2002). Los sordos defienden su silencio. El País, 14 abril 2002.

Allué M. (2003). DisCapacitados. La reivindicación de la igualdad en la diferencia. Barcelona, Bellaterra.

Anderson Y. (1994). Comment on Turner. Sign Language Studies, 83, 127-131.

Bahan B. (1994). Comment on Turner. Sign Language Studies, 84, 241-249. 


\section{perifèria}

Número 18, junio 2013

http://revistes.uab.cat/periferia

Bahan B., Bauman H-D. L. (2000). Audism: Toward a postmodern theory of deaf studies. En: Deaf Studies VI conference. Orlando, Florida.

Baker C., Paden C. (1978). ASL: A look at its history, structure and community. Silver Spring: TJ Publishers.

Batterbury S., Ladd P., Gulliver M. (2007). Sign Language Peoples as indigenous minorities: implications for research and policy. Environment and Planning $A, 39$, $12,2899-2915$.

Bauman H-D. L. (2004). Audism: Exploring the Metaphysics of Oppression. Journal of Deaf Studies and Deaf Education, 9, 2, 239-246.

Bauman H-D. L., ed. (2008a). Open Your Eyes. Deaf Studies Talking. Minneapolis:University of Minnesota Press.

Berger P.L, Luckmann T. (2001). La construcción social de la realidad. Buenos Aires: Amorrortu ediciones.

Cándido (2002) La sordera como identidad. ABC, 15 abril 2002.

Cedillo P. (2004). Háblame a los ojos. Barcelona: Ediciones Octaedro.

Díaz-Estébanez M.E., et al. (1996). Las personas sordas y su realidad social: un estudio descriptivo. Madrid: Ministerio de Educación y Ciencia.

Fernández-Viader M.P, Pertusa E., coords. (2004). El Valor de la mirada: sordera y educación. Barcelona: Publicacions i Edicions Universitat de Barcelona.

Goffman I. (2006). Estigma: la identidad deteriorada. Buenos Aires: Amorrortu ediciones.

González A. (2009). La dicotomía emic/etic. Historia de una confusión. Rubí: Anthropos Editorial.

González E. (2002). Sordos por decisión materna. El País, 9 abril 2002.

Humphries T. (1977). Communicating across cultures (deaf-/hearing) and language learning. Cincinnati, $\mathrm{OH}$ : Union Institute and University. Doctoral dissertation.

Johnson R., Erting C. (1989). Etnicity and socialization in classroom for deaf 


\section{perifèria}

Número 18, junio 2013

http://revistes.uab.cat/periferia

children. En: Lucas C., comp. (1989) The sociolinguistics of the Deaf Community. San Diego: Academic Press.

Kyle J., Allsop L. (1982). Deaf People and Society. Bristol: School of Education.

Kyle J. (1990). The Deaf Community: Culture, Custom and Tradition. En: Prillwitz S., Wollhaber T., eds. (1990). Sign Language Research and Application. Hamburg: Signum Press.

Ladd P. (2003). Understanding Deaf Culture. In Search of Deafhood. Toronto: Multilingual Matters.

Lane H. (1992). The Mask of Benvolence: Disabling the Deaf Community. New York: Knopf.

Leigh W.I. (2009). A Lens on Deaf Identities. New York:Oxford University Press.

Martínez C., et al. (2007). Sordo iy qué!. Madrid: LoQueNoExiste.

Mcllroy G., Storbeck C. (2011). Development of Deaf Identity: An Etnographic Study. Journal of Deaf Studuies and Deaf Education, Jun 20, 1-18.

Mills M. (2002). I'm happy my child is deaf. The Guardian, 9 abril 2002.

Morales A.M. (2009). La ciudadanía desde la diferencia: reflexiones en torno a la comunidad sorda. Revista Latinoamericana de Educación Inclusiva, 3, 2, 125-141.

Moreno A. (2000). La Comunidad Sorda: aspectos psicológicos y sociológicos. Madrid: Fundación CNSE.

Mundy L. (2002). A World of Their Own. The Washington Post, 31 marzo 2002.

Nover S. (1995). Language and English in deaf education. En: Lucas C., ed. (1995) Sociolinguistics in deaf communities. Washington, DC: Gallaudet University Press, 109-163.

Padden C., Humphries T. (1988). Deaf in America: Voices from a Culture. Cambridge: Harvard University Press.

Padden C. (1989). The Deaf Comunity and the Culture of Deaf people. En: Wilcox S., ed. (1989). American Deaf Culture. Silver Spring: Linstok Press. 


\section{perifèria \\ Número 18, junio 2013 \\ http://revistes.uab.cat/periferia}

Pinedo F.J. (1981). El sordo y su mundo. Madrid: CNSE.

Pinedo F.J. (1989). Una voz para un silencio. Madrid: CNSE.

Pino F., Monterde I. (2001). Sociología de las personas sordas. En: Minguet A., coord. (2001) Rasgos sociológicos y culturales de las Personas Sordas. Valencia: FESORD， 197-248.

Sandel M. (2007). Contra la perfección. La ética en la era de la ingeniería genética. Barcelona: Marbot Ediciones.

Schein J.D. (1968). The Deaf Community. Studies in the Social Psychology of Deafness. Washington, DC.: Gallaudet College Press.

Stokoe W. (1960). Sign language structure: An outline of the visual communication system of the American Deaf. Studies in Linguistics, Ocassional paper.

Stokoe W., Casterline D, Groneberg C. (1965). A Dictionary of American Sign Language on Linguistic Principles. Silver Spring: Linstok Press.

Storch de Gracia J.G. (1998). El nombre de nuestra lengua. Comunicación al I Congreso Ibero-Americano de Educación Bilingüe para Sordos (Lisboa, 6-10 julio 1998), julio 1998, policopiado, 35 páginas.

Sortch de Gracia J.G. (2008). Las lenguas de señas ante el Derecho civil. Revista General de Legislación y Jurisprudencia, 2, abril-junio, 259-302.

Torres D.A. (2011). Estrategias y recursos para el desarrollo de competencias en el niño Sordo. Xalapa, México: Kofo Ediciones.

Turner G. (1994). How is Deaf Culture: Toward a revised notion of fundamental concept. Sing Language Studies, 83, 103-126.

Villalba A. (2001). Sordera. En: Minguet A., coord. (2001) Rasgos sociológicos y culturales de las Personas Sordas. Valencia: FESORD, 5-59.

Woodward J. (1972.) Implications for sociolinguistics research among the deaf. Sign Language Studies, 1, 1-7. 